\title{
Dynamic expression of the LAP family of genes during early development of Xenopus tropicalis
}

\author{
YANG QiuTan $^{1,2 \dagger}$, LV XiaoYan ${ }^{1 \dagger}$, KONG QingHua ${ }^{2}$, LI ChaoCui ${ }^{2}$, \\ ZHOU Qin ${ }^{1 *} \&$ MAO BingYu ${ }^{2 *}$ \\ ${ }^{1}$ Core Facility of Genetically Engineered Mice, State Key Laboratory of Biotherapy, West China Hospital, \\ Sichuan University, Chengdu 610041, China; \\ ${ }^{2}$ CAS-Max Planck Junior Scientist Group, State Key Laboratory of Genetic Resources and Evolution, \\ Kunming Institute of Zoology, Chinese Academy of Sciences, Kunming 650223, China
}

Received March 7, 2011; accepted March 28, 2011

\begin{abstract}
The leucine-rich repeats and PDZ (LAP) family of genes are crucial for the maintenance of cell polarity as well as for epithelial homeostasis and tumor suppression in both vertebrates and invertebrates. Four members of this gene family are known: densin, erbin, scribble and lano. Here, we identified the four members of the LAP gene family in Xenopus tropicalis and studied their expression patterns during embryonic development. The Xenopus LAP proteins show a conserved domain structure that is similar to their homologs in other vertebrates. In Xenopus embryos, these genes were detected in animal cap cells at the early gastrula stage. At later stages of development, they were widely expressed in epithelial tissues that are highly polar in nature, including the neural epithelia, optic and otic vesicles, and in the pronephros. These data suggest that the roles of the Xenopus LAP genes in the control of cell polarity and morphogenesis are conserved during early development. Erbin and lano show similar expression patterns in the developing head, suggesting potential functional interactions between the two molecules in vivo.
\end{abstract}

\section{Xenopus tropicalis, LAP gene family, Densin, Erbin, Scrib, Lano, expression pattern}

Citation: Yang Q T, Lv X Y, Kong Q H, et al. Dynamic expression of the LAP family of genes during early development of Xenopus tropicalis. Sci China Life Sci, 2011, 54: 897-903, doi: 10.1007/s11427-011-4224-4

The LAP (leucine-rich repeats and PDZ) proteins are evolutionarily conserved adaptor proteins involved in the control of cell polarity and proliferation [1]. In vertebrates, there are four LAP proteins: Densin-180 (Densin), Erbin (erbb2-interacting protein), Scribble (Scrib), and Lano [2,3]. Each of these proteins contains a LRR (the leucine-rich repeat) domain at the N-terminal, two LAPSD (LAP-specific domain) motifs and four, one or no PDZ (PSD-95/Dlg-A/ZO-1) domains in the C-terminal region. The LRR domain, consisting of 16 leucine-rich repeats of about 20 to 30 amino acids, guides cellular basolateral locations of the LAP protein and has

\footnotetext{
$\dagger$ Contributed equally to this work

*Corresponding author (email: pkdzhou@126.com; mao@mail.kiz.ac.cn)
}

diverse functions [4,5]. The LAPSD domains are LRR-like domains conserved only in LAP proteins [3]. The PDZ domain, consisting of six $\beta$-strands and two $\alpha$-helices, is believed to mediate protein-protein interactions.

Densin (also known as leucine rich repeat containing 7, Lrrc7) was originally identified from rat forebrain and was the first LAP protein to be characterized [6]. In mammals, Densin interacts with tissue specific proteins to participate in the formation of synaptic junctions in the brain and in the slit diaphragm in the glomus of kidney [7-9]. Erbin interacts with various proteins and has been shown to be important for neural development and epithelial homeostasis $[10,11]$. Scrib is well known as a polarity protein and a tumor suppressor in both vertebrates and invertebrates 
$[1,2,12]$. The Scrib complex is essential for correct implementation of apical/basal polarity $[2,5,13]$ and participates in the Wnt/PCP signaling pathway with Vangl2 to affect many developmental events [14-16]. Lano (also known as leucine rich repeat containing 1, Lrrc1) was the last member of the LAP protein family to be reported. Lano directly interacts with the PDZ domains of hDLG (human disc large) and Erbin in epithelial cells [17].

Because of their essential roles in the regulation of tissue polarity, the $L A P$ genes are widely expressed during embryonic development and organogenesis. In zebrafish, scrib is maternally detected, and its expression is restricted in the brain at later stages [18]. In mouse, Scrbl, the ortholog of $S c r i b$, is widely expressed in epithelial tissues including the neuroepithelium, heart, liver, kidney, esophagus, and lung epithelia during embryonic and fetal development [19]. However, systematic analyses of the developmental expression patterns of the $L A P$ genes in vertebrates are lacking. Here, we identified the four members of the $L A P$ gene family in Xenopus tropicalis and studied their embryonic expression patterns by whole mount in situ hybridization (WMISH). The widespread and overlapping expression of the $L A P$ genes in the epithelial tissues likely suggest their conserved roles in the control of tissue polarity.

\section{Materials and methods}

\subsection{Characterization and phylogenetic analysis of the LAP genes}

BLAST searches against the Xenopus tropicalis genome [20] were carried out using the human LAP proteins as query sequences. The $X$. tropicalis $(X t)$ EST databases from the Sanger Center EST collection (http://www.sanger.ac.uk/ Projects/X_tropicalis/) and from the DNA Data Bank of Japan (http://blast.ddbj.nig.ac.jp/top-e.html) were also used as the targets. EST assembly was performed using the Lasergene software package. For Xt-scrib, an EST clone (IMAGE: 5379424) was completely sequenced and the sequence was submitted to GenBank (GenBank ID: JN542544). The phylogenetic tree was constructed as described earlier [21].

\subsection{Semi-quantitative RT-PCR assay}

Total RNA was prepared from embryos at selected stages of development using TRIZOL reagent (Invitrogen, Carlsbad, CA, USA) according to the manufacturer's protocol. First-strand cDNA was synthesized from $1 \mu \mathrm{g}$ of total RNA with random primers using the RevertAid $\mathrm{H}$ minus first strand cDNA Synthesis kit (Fermentas, Vilnius, Lithuania).

Primers used for PCR were as follows: $X t$-scrib: sense primer: 5'-CTCAAGGTGGATCAGAACCGT-3'; antisense primer: 5'-CCAACCACAGAGCTTTCAGGT-3'; Xt-densin: sense primer: 5'-CCATAGTTGAAGCCAGCGT-3'; antisense primer: 5'-CCTAGATCAAGCCTTCTCCAG-3'; Xterbin: sense primer: 5'-GAGTGTCAGGAAGAGGTT-3'; antisense primer: 5'-GTGAGAGTAGTCAAGAGAGGT- 3'; Xt-lano: sense primer: 5'-GCACTCCAAGTAGCTGAT-3'; antisense primer: 5'-CTTCAATCCACTGATCTCTTC-3'; H4 was used as an internal control [22].

\subsection{Whole-mount in situ hybridization and sectioning of embryos}

$X$. tropicalis embryos were obtained by in vitro fertilization and cultured at $28.5^{\circ} \mathrm{C}$ in $0.1 \times \mathrm{MBS}$. For in situ hybridization, fragments of $X t$-scrib (nucleotides 51-764 of GenBank ID: JN542544), Xt-densin (nucleotides 1724-2235 of GenBank ID: NM_001079126), Xt-erbin (nucleotides 42-522 of GenBank ID: XM_002934188) and Xt-lano (nucleotides 1091-1939 of GenBank ID: NM_204055) in the less conserved (most gene-specific) regions, were cloned for probe preparation. Whole-mount in situ hybridization was carried out as described [21]. Embryos were bleached in a solution of $0.5 \times \mathrm{SSC}, 5 \%$ formamide, $1 \% \mathrm{H}_{2} \mathrm{O}_{2}$. For sectioning, embryos were embedded in paraffin and sectioned to a thickness of $25 \mu \mathrm{m}$.

\section{Results}

\subsection{Characterization and phylogenetic analysis of $X t$-LAPs}

The human LAP protein sequences were used to BLAST against the NCBI transcript databases and against the $X$. tropicalis genome. The full-length Xt-densin and Xt-lano mRNA sequences were identified in GenBank (IDs: NM_001079126 and NM_204055, respectively). However, the predicted $X t$-Densin protein contained an incomplete PDZ domain. The 3' sequence of an EST clone (GenBank ID: CX842329) was found to encode the full-length PDZ domain of $X t$-Densin. Analysis of the corresponding genomic sequence revealed that the NM_001079126 sequence most likely represented a short splicing isoform lacking 17 nucleotides in the second last coding exon which resulted in a pre-mature stop codon. In mouse, densin also undergoes extensive alternative splicing and isoforms lacking the PDZ domain that might have different protein-interaction abilities have been reported [23]. The predicted sequence of Xt-erbin (GenBank ID: XM_002934188) was used. For $X t$-scrib, an EST sequence (GenBank ID: BQ523607) that corresponded to the $5^{\prime}$ untranslated region was found. This clone (IMAGE: 5379424) which was completely sequenced contains a full open reading frame of 2928 nucleotides.

The deduced $X t$-Densin, $X t$-Erbin, $X t$-Scrib and $X t$-Lano proteins contain 1445, 1359, 976 and 524 amino acids respectively. The $X t$-LAP proteins exhibit typical LAP protein 
domain structures (Figure 1A) that includes an N-terminal LRR domain, two LAP-specific domains (LAPSDa and LAPSDb [3]), and either PDZ domains or a PDZ binding site at the C-terminal. The conserved domains, especially the LAPSDa and LAPSDb domains, show high similarity to previously characterized LAP proteins in other vertebrates (data not shown). However, the deduced $X t$-Scrib protein contains only two PDZ domains (Figure 1A), unlike the Scrib proteins in other vertebrates that all contain four PDZ domains. This observation indicates that the $X t$-Scrib clone that we identified might represent a short isoform (Figure 1A). Phylogenetic analysis revealed high conservation of the LAP proteins in vertebrates (Figure 1B) and supports the view that the four $L A P$ genes in vertebrates originated through gene duplication during vertebrate origin from two prototypes in protostomes [3].

A

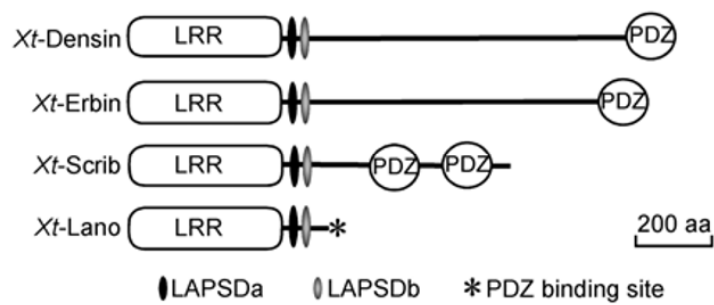

B

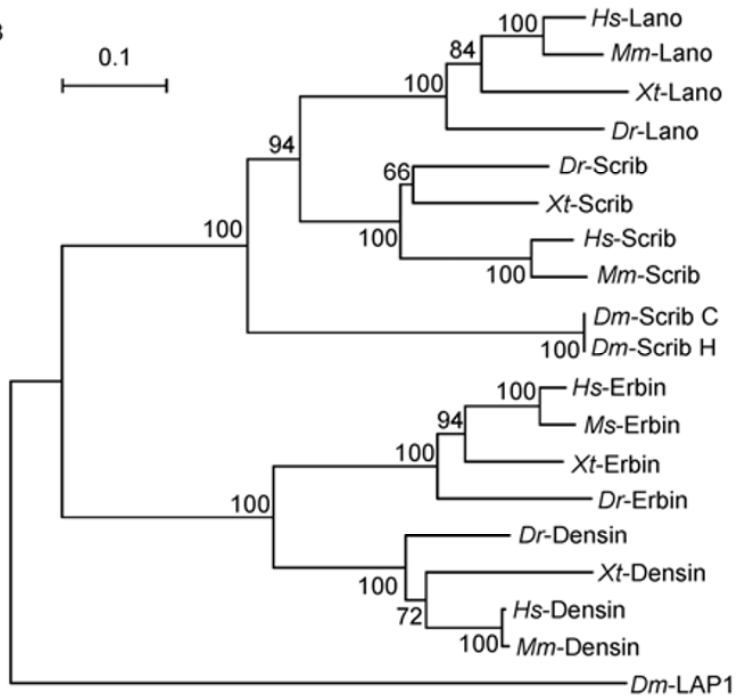

Figure 1 The domain structure and phylogenetic analysis of Xenopus tropicalis LAP proteins. A, Domain structure of the X. tropicalis LAP proteins. B, Phylogenetic analysis of Homo sapiens $(H s)$, Mus musculus $(\mathrm{Mm})$, Xenopus tropicalis $(\mathrm{Xt})$, Danio rerio $(\mathrm{Dr})$ and Drosophila melanogaster $(D m)$ LAP family of proteins. ClustalW alignment, the Poisson correction model, and a bootstrap test (1000 replicates) were used for neighbor-joining (NJ) tree construction. The GenBank sequences used for construction of the tree were $H s$-Lano (NP_060684), Mm-Lano (NP_001139520), $X t$-Lano (NP_989386), $D r$-Lano (NP_001013463), Hs-Scrib (NP_056171), $M m$-Scrib (NP_598850), Dr-Scrib (NP_001007176), Dm-Scrib C (NP_733156), Dm-Scrib H (NP_001014670), $H s$-Erbin (NP_061165), $M m$-Erbin (NP 001005868), Dr-Erbin (NP 001073500), Hs-Densin (NP_065845), $M m$-Densin (NP_001074827), Xt-Densin (NP_001072594), $D r$-Densin (XP_001920812) and Dm-LAP1 (NP_611007).

\subsection{Temporal expression of $X$. tropicalis $L A P$ genes}

To investigate the temporal expression patterns of the $L A P$ genes during $X$. tropicalis development, a series of RT-PCR analysis was carried out (Figure 2). All four genes were detected maternally and all showed different temporal expression patterns during development. The expression of $X t$-densin remained relatively weak till about stage 30 and then it markedly increased at stage 36. Xt-erbin and Xt-lano were expressed strongly from the egg to the early neurula stage and became weak between stages 14-25. Their expression again went up at about stage 30. Xt-scrib was expressed at a steady level throughout the stages examined.

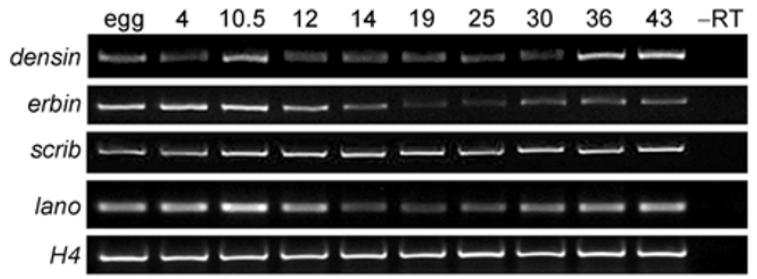

Figure 2 RT-PCR analysis of the developmental expression of the LAP genes in Xenopus tropicalis. RT, the product of an RT reaction without reverse transcriptase was used as a template for the negative control.

\subsection{Spatial expression of $L A P$ genes during $X$. tropicalis early development}

At the cleavage stage, $X t$-densin was expressed in the animal blastomeres (Figure 3A) and at the late blastula stage $X t$-densin was detected in the inner layer cells of the animal cap (Figure 3B). At the gastrula stages, compared to the sense probe control, a weak ubiquitous expression signal was detected in the ectoderm (Figure 3C and D). At stage $16, X t$-densin was detected in the neural plate and paraxial mesoderm (Figure 3E). From stages 26-32 (Figure 3F-H), $X t$-densin was strongly expressed in the brain, eyes, otic vesicles, somites, and branchial arches and in the pronephros, it was strongly expressed in the pronephric tubules and duct (Figure 3F and $\mathrm{H}$ ). Transverse sections revealed that the expression of $X t$-densin was strong in the developing pronephric tubules (Figure 3I). At stage 36, it was widely expressed in the brain, eyes, otic vesicles, spinal cord, pronephros, somites, and branchial arches (Figure 3J).

At the 4-cell stage, Xt-erbin transcripts were detected at the animal poles of the blastomeres (Figure 4A) and at the early gastrula stage, it was weakly detected in the inner cell layer of the animal cap (Figure 4B). At gastrula stages, it was weakly ubiquitously expressed in the ectoderm compared with in the sense probe control (Figure 4C and D). At stage 16, Xt-erbin was expressed in the cement gland, forebrain, hindbrain and weakly in the spinal cord, and paraxial mesoderm (Figure 4E). At tail bud and tadpole stages, $X t$-erbin was expressed strongly in the brain, eyes, otic vesicles, cement gland and weakly in the entire spinal cord 

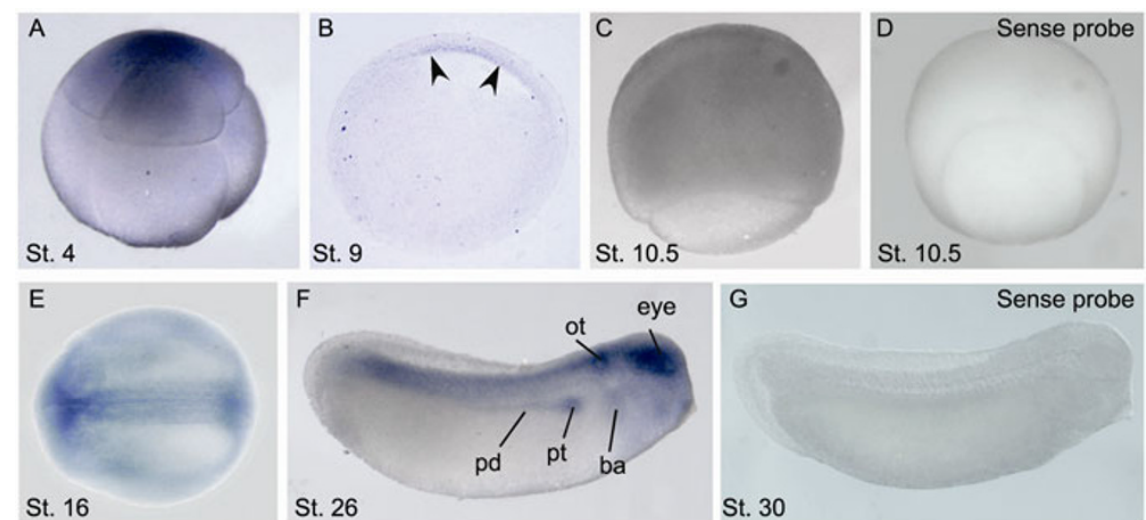

\section{St. 16}

St. 26
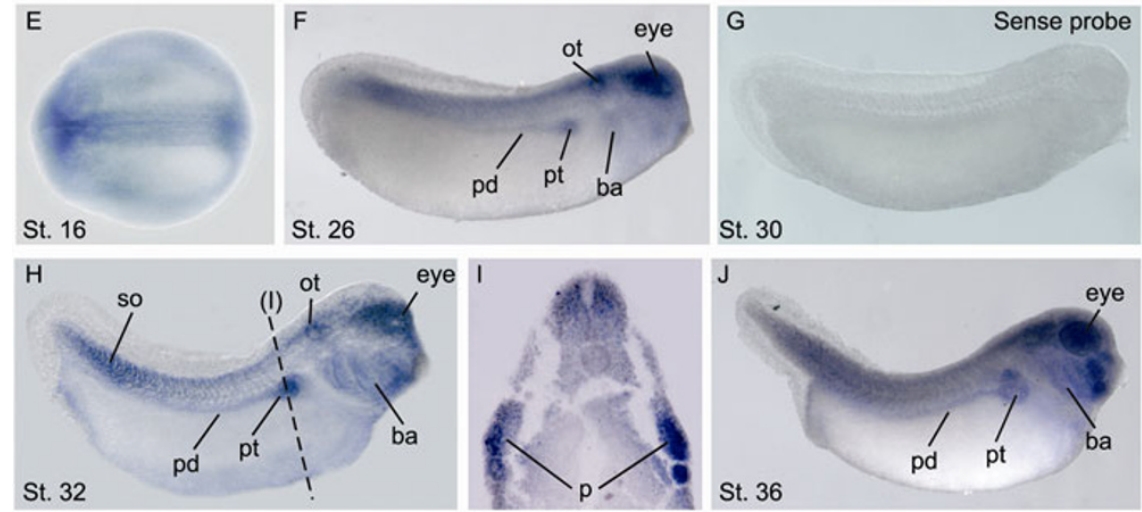

Figure 3 Embryonic expression of Xt-densin at various stages of development. A, Stage 4, lateral view with the animal pole to the top. B, Stage 9, a section of an embryo with animal pole to the top. The arrowheads indicate the expression in the inner layer of the animal cap. C, Stage 10.5, lateral view with vegetal pole to the bottom. $\mathrm{D}$, Stage 10.5 sense probe control, lateral view with vegetal pole to the bottom. E, Stage 16 , dorsal view with anterior to the right. F, Stage 26, lateral view with anterior to the right. G, Stage 30 sense probe control, lateral view with anterior to the right. H, Stage 32 , lateral view with anterior to the right. I, Stage 32, a section through the dotted line in H showing the expression of densin in the pronephros. J, Stage 36 , lateral view with anterior to the right. Abbreviations: ba, branchial arches; ot, otic vesicle; p, pronephros; pd, pronephric duct; pt, pronephric tubules; so, somite.
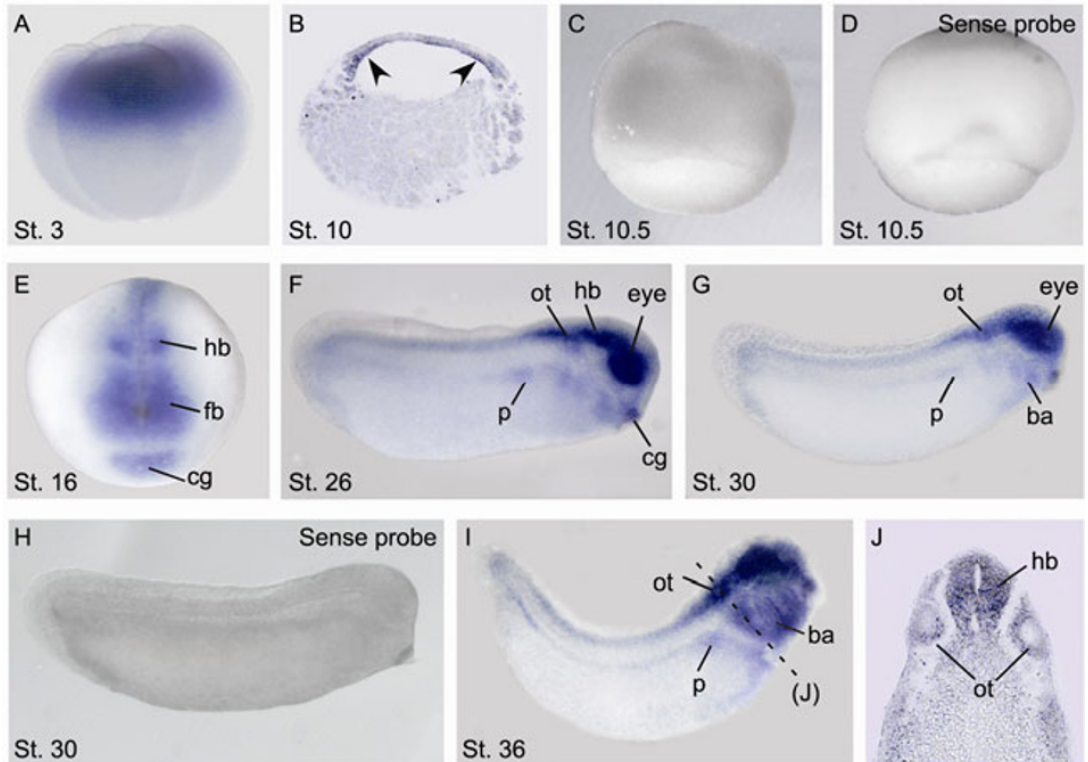

St. 30

St. 30

St. 36

Figure 4 Embryonic expression of Xt-erbin at various stages of development. A, Stage 3, lateral view with animal pole to the top. B, Stage 10, a section of an embryo with animal pole to the top. The arrowheads indicate the expression in the inner layer cells of the animal cap. C, Stage 10.5 , lateral view with vegetal pole to the bottom. D, Stage 10.5 sense probe control, lateral view with vegetal pole to the bottom. E, Stage 16 , frontal view with dorsal to the top. F, Stage 26, lateral view with anterior to the right. G, Stage 30, lateral view with anterior to the right. H, Stage 30 sense probe control, lateral view with anterior to the right. I, Stage 36, lateral view with anterior to the right. J, A section through the dotted line in I showing the expression of erbin in the otic vesicles and hindbrain. Abbreviations: ba, branchial arches; cg, cement gland; fb, forebrain; hb, hindbrain; ot, otic vesicle; $p$, pronephros.

(Figure 4F-J). At these stages, Xt-erbin was also detected in the branchial arches and weakly in the pronephros (Figure 4F-J).

At the cleavage stage, $X t$-scrib transcripts were detected in the blastomeres at the animal pole (Figure 5A) and at gastrula stages they were strongly detected in the ectoderm (Figure 5B and C). At neurula stages, Xt-scrib was strongly expressed in the neural plate and paraxial mesoderm (Figure 

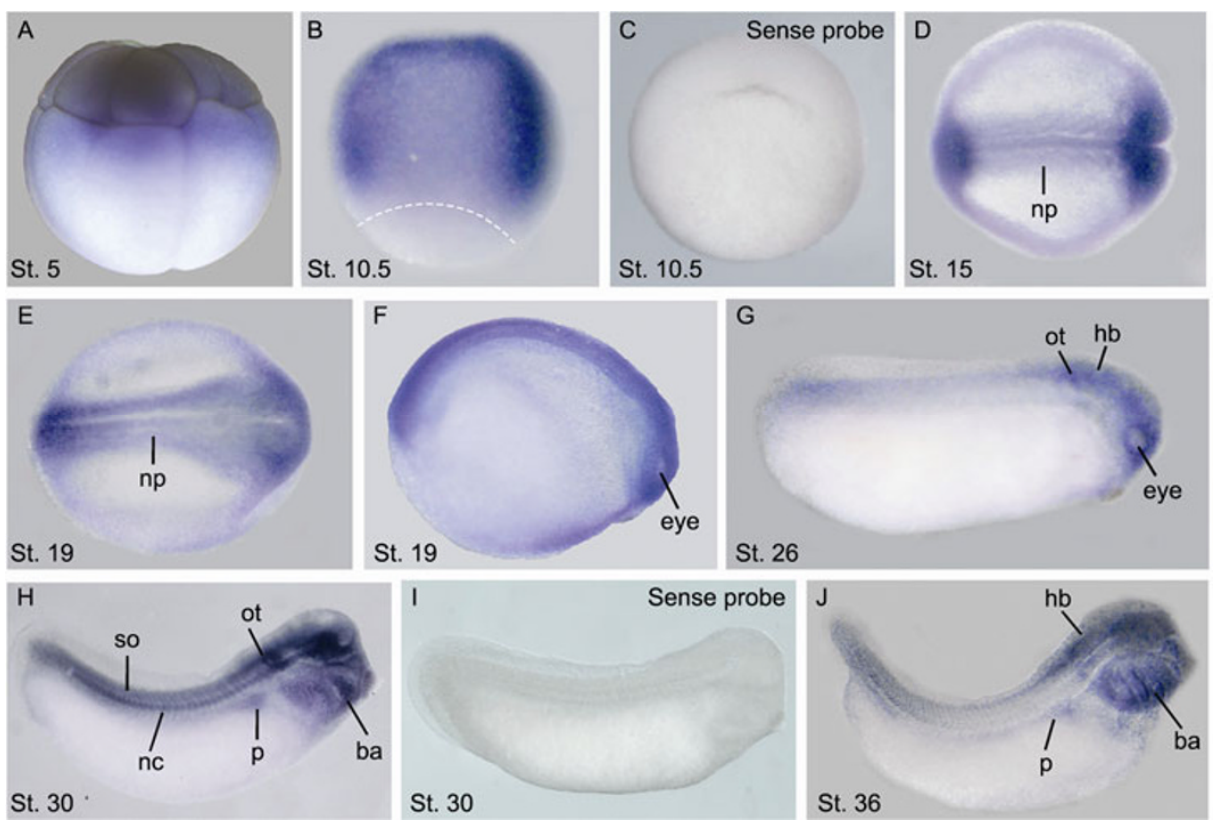

Figure 5 Embryonic expression of Xt-scrib at various stages of development. A, Stage 5, lateral view with animal pole to the top. B, Stage 10.5, lateral view with vegetal pole to the bottom. The broken white line outlines the border of the blastopore. C, Stage 10.5 sense probe control, vegetal view. D, Stage 15 , dorsal view with anterior to the right. E, Stage 19, dorsal view with anterior to the right. F, Stage 19, lateral view with anterior to the right. G, Stage 26, lateral view with anterior to the right. $\mathrm{H}$, Stage 30 , lateral view with anterior to the right. I, Stage 30 sense probe control, lateral view with anterior to the right. J, Stage 36, lateral view with anterior to the right. Abbreviations: ba, branchial arches; hb, hindbrain; nc, notochord; np, neural plate; ot, otic vesicle; p, pronephros; so, somite.

$5 \mathrm{D}$ and $\mathrm{E})$, as was reported previously in mouse and zebrafish [18,19]. From stage 26, Xt-scrib was expressed in the developing eyes and otic vesicles (Figure 5G) and at tailbud stages, it was widely expressed in the central nervous system (CNS), including the brain, spinal cord, eyes, and otic vesicles (Figure 5H-J). At stage 30, Xt-scrib was also expressed strongly in the notochord, somites, and branchial arches and weakly in the developing pronephros (Figure $5 \mathrm{H}$ ) and at stage 36, it was expressed strongly in the hindbrain region and branchial arches (Figure 5J).

At the 4-cell stage, Xt-lano was detected at the animal poles of the blastomeres (Figure 6A). At the early gastrula stage, Xt-lano mRNA was weakly detected in the ectoderm with no clear pattern (Figure 6B-D) and at early neurula stages, its expression was relatively weak and no clear pattern could be detected (data not shown). At stage 26, $X t$-lano was expressed in the cement gland, eyes, tail bud, and weakly in the pronephros anlage (Figure 6E) and at stage 30, it was also detected in the branchial arches and developing otic vesicles (Figure 6F and G). Xt-lano expression continued in these domains at stage 36 and was also detected in the developing heart (Figure 6H and I).

\section{Discussion}

LAP family genes have been shown to be involved in the regulation of cell and tissue polarity and morphogenesis $[5,14,24,25]$. For example, Scrib is involved in the
Wnt/PCP pathway [16] and disruption of Scrib causes failure of gastrulation in zebrafish [18] and severe neural tube and heart defects in mouse $[15,19]$. In zebrafish, mutation of scrib also blocks the migration of nVII motor neurons [18]. Here, we have shown that the Xt-LAP genes are widely expressed in $X$. tropicalis epithelial tissues with high polarity that include the neural epithelia, optic and otic vesicles, and pronephros. The largely overlapping expression patterns of the four genes suggest partially redundant roles for these genes. At early gastrula stages, the Xt-LAP genes were widely expressed in the animal cap cells, suggesting that they might be involved in the regulation of epithelial polarity and gastrulation. The widespread expression of $X t-L A P$ genes in the CNS suggests their possible conserved roles in neural development. All four genes are co-expressed in the developing pronephric tubules and duct. In the metanephros of mammals, densin is expressed in the glomerular podocytes and was shown to interact with Nephrin at the slit diaphragm [7]. In zebrafish, Scrib is also required for pronephros development [26]. Thus, we speculate that the $L A P$ genes might play overlapping roles in the control of epithelial polarity and morphogenesis of the pronephros in Xenopus.

Lano, which does not contain the PDZ domain, was the last member of the LAP protein family to be reported. Its developmental functions remain unexplored. The wide expression of Xt-lano in the epithelial tissues of $X$. tropicalis suggests that it might have a similar role in the control of tissue polarity as the other LAP genes. Lano contains a PDZ 

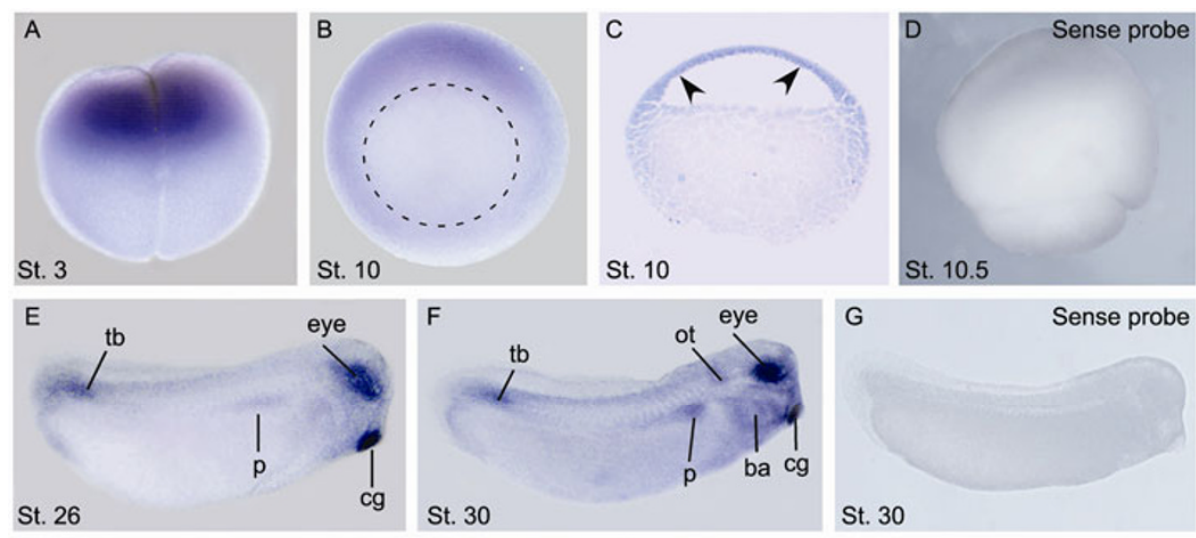

Sense probe

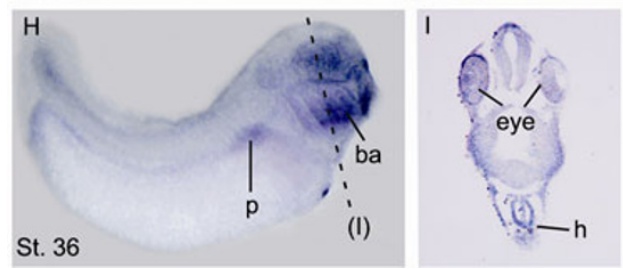

Figure 6 Embryonic expression of Xt-lano at various stages of development. A, Stage 3, lateral view with animal pole to the top. B, Stage 10, vegetal view. The broken black line indicates the border of the blastopore. C, Stage 10, a section of the embryo with animal pole to the top. The arrowheads indicate the expression in the animal cap. D, Stage 10.5 sense probe control, lateral view with animal pole to the top. E, Stage 26 , lateral view with anterior to the right. F, Stage 30, lateral view with anterior to the right. G, Stage 30 sense probe control, lateral view with anterior to the right. H, Stage 36 , lateral view with anterior to the right. I, A section through the dotted line in H showing the expression of lano in the heart. Abbreviations: ba, branchial arches; cg, cement gland; h, heart; ot, otic vesicle; p, pronephros; tb, tail bud.

binding site at its C-terminal and may participate in the regulation of tissue polarity through its interaction with PDZ domains in other proteins. Lano has been shown to interact with the PDZ domain of Erbin but not with Scrib and Densin [17]. Interestingly, Xt-lano and Xt-erbin show similar expression patterns in the developing head (Figures 4 and 6). Both of these genes were strongly expressed in the cement gland where densin and scrib were not detected, supporting the possibility of functional interactions between the two molecules. In $X$. laevis, lano (SCRPI) was isolated in a screen for anterior neuroectoderm expressed genes and was shown to be weakly ubiquitously expressed in the neural plate at the neurula stage [27].

We thank Dr. Chen YongLong for providing some of the Xenopus tropicalis embryos. This work was supported by the Key State Research Program from Ministry of Science and Technology of China (Grant No. 2007CB947201) and the State Key Laboratory of Genetic Resources and Evolution.

1 Bryant P J, Huwe A. LAP proteins: what's up with epithelia? Nat Cell Biol, 2000, 2: E141-143

2 Bilder D, Perrimon N. Localization of apical epithelial determinants by the basolateral PDZ protein Scribble. Nature, 2000, 403: 676-680

3 Santoni M J, Pontarotti P, Birnbaum D, et al. The LAP family: a phylogenetic point of view. Trends Genet, 2002, 18: 494-497

4 Kajava A V. Structural diversity of leucine-rich repeat proteins. J Mol Biol, 1998, 277: 519-527

5 Legouis R, Gansmuller A, Sookhareea S, et al. LET-413 is a basolateral protein required for the assembly of adherens junctions in Caenorhabditis elegans. Nat Cell Biol, 2000, 2: 415-422
6 Apperson M L, Moon I S, Kennedy M B. Characterization of densin180 , a new brain-specific synaptic protein of the O-sialoglycoprotein family. J Neurosci, 1996, 16: 6839-6852

7 Ahola H, Heikkila E, Astrom E, et al. A novel protein, densin, expressed by glomerular podocytes. J Am Soc Nephrol, 2003, 14: 1731-1737

8 Izawa I, Nishizawa M, Ohtakara K, et al. Densin-180 interacts with delta-catenin/neural plakophilin-related armadillo repeat protein at synapses. J Biol Chem, 2002, 277: 5345-5350

9 Quitsch A, Berhöster K, Liew C W, et al. Postsynaptic shank antagonizes dendrite branching induced by the leucine-rich repeat protein Densin-180. J Neurosci, 2005, 25: 479-487

10 Borg J P, Marchetto S, Le Bivic A, et al. ERBIN: a basolateral PDZ protein that interacts with the mammalian ERBB2/HER2 receptor. Nat Cell Biol, 2000, 2: 407-414

11 Tao Y, Dai P, Liu Y, et al. Erbin regulates NRG1 signaling and myelination. Proc Natl Acad Sci USA, 2009, 106: 9477-9482

12 Zhan L, Rosenberg A, Bergami K C, et al. Deregulation of scribble promotes mammary tumorigenesis and reveals a role for cell polarity in carcinoma. Cell, 2008, 135: 865-878

13 Zeitler J, Hsu C P, Dionne H. et al. Domains controlling cell polarity and proliferation in the Drosophila tumor suppressor Scribble. J Cell Biol, 2004, 167: 1137-1146

14 Montcouquiol M, Rachel R A, Lanford P J, et al. Identification of Vang12 and Scrb1 as planar polarity genes in mammals. Nature, 2003, 423: $173-177$

15 Phillips H M, Rhee H J, Murdoch J N, et al. Disruption of planar cell polarity signaling results in congenital heart defects and cardiomyopathy attributable to early cardiomyocyte disorganization. Circ Res, 2007, 101: 137-145

16 Vervenne H B, Crombez K R, Lambaerts K, et al. Lpp is involved in Wnt/PCP signaling and acts together with Scrib to mediate convergence and extension movements during zebrafish gastrulation. Dev Biol, 2008, 320: 267-277

17 Saito H, Santoni M J, Arsanto J P, et al. Lano, a novel LAP protein directly connected to MAGUK proteins in epithelial cells. J Biol 
Chem, 2001, 276: 32051-32055

18 Wada H, Iwasaki M, Sato T, et al. Dual roles of zygotic and maternal Scribble1 in neural migration and convergent extension movements in zebrafish embryos. Development, 2005, 132: 2273-2285

19 Murdoch J N, Henderson D J, Doudney K, et al. Disruption of scribble (Scrb1) causes severe neural tube defects in the circletail mouse. Hum Mol Genet, 2003, 12:87-98

20 Hellsten U, Harland R M, Gilchrist M J, et al. The genome of the Western clawed frog Xenopus tropicalis. Science, 2010, 328: 633-636

21 Zhao S, Jiang H, Wang W, et al. Cloning and developmental expression of the Xenopus Nkx6 genes. Dev Genes Evol, 2007, 217: 477-483

22 Knöchel S, Dillinger K, Köster M, et al. Structure and expression of Xenopus tropicalis BMP-2 and BMP-4 genes. Mech Dev, 2001, 109: $79-82$
23 Jiao Y, Robison A J, Bass M A, et al. Developmentally regulated alternative splicing of densin modulates protein-protein interaction and subcellular localization. J Neurochem, 2008, 105: 1746-1760

24 Jaulin-Bastard F, Arsanto J P, Le Bivic A, et al. Interaction between Erbin and a Catenin-related protein in epithelial cells. J Biol Chem, 2002, 277: 2869-2875

25 Ohtakara K, Nishizawa M, Izawa I, et al. Densin-180, a synaptic protein, links to PSD-95 through its direct interaction with MAGUIN-1. Genes Cells, 2002, 7: 1149-1160

26 Skouloudaki K, Puetz M, Simons M, et al. Scribble participates in Hippo signaling and is required for normal zebrafish pronephros development. Proc Natl Acad Sci USA, 2009, 106: 8579-8584

27 Takahashi N, Tochimoto N, Ohmori S Y, et al. Systematic screening for genes specifically expressed in the anterior neuroectoderm during early Xenopus development. Int J Dev Biol, 2005, 49: 939-951

Open Access This article is distributed under the terms of the Creative Commons Attribution License which permits any use, distribution, and reproduction in any medium, provided the original author(s) and source are credited. 